\title{
Downsizing a Great Observatory: Reinventing Spitzer in the Warm Mission
}

\author{
Lisa J. Storrie-Lombardi ${ }^{* a}$ and Suzanne R. Dodd ${ }^{\mathrm{b}}$ \\ ${ }^{a}$ Spitzer Science Center, California Institute of Technology, M/S 314-6, \\ 1200 E. California Blvd., Pasadena, CA 91125 \\ ${ }^{\mathrm{b} J e t}$ Propulsion Laboratory, 4800 Oak Grove Drive, Pasadena, CA 91109
}

\begin{abstract}
The Spitzer Space Telescope transitioned from the cryogen mission to the IRAC warm mission during 2009. This transition involved changing several areas of operations in order to cut the mission annual operating costs to $1 / 3$ of the cryogen mission amount. In spite of this substantial cut back, Spitzer continues to have one of the highest science return per dollar ratio of any of NASA's extended missions. This paper will describe the major operational changes made for the warm mission and how they affect the science return. The paper will give several measures showing that warm Spitzer continues as one of the most scientifically productive mission in NASA's portfolio. This work was performed at the California Institute of Technology under contract to the National Aeronautics and Space Administration.
\end{abstract}

Keywords: Spitzer Space Telescope, Operations, Extended Mission

\section{INTRODUCTION}

The Spitzer Space Telescope, NASA's Great Observatory for infrared astronomy, was launched 25 August $2003^{[1]}$. It is the fourth and final element in NASA's Great Observatories program that includes the Hubble Space Telescope (1990), the Compton Gamma-Ray Observatory (1991-2000), and the Chandra X-Ray Observatory (1999). Note that, until 2003 December, Spitzer was known as the Space Infrared Telescope Facility (SIRTF). Spitzer provides sensitivity that is almost three orders of magnitude greater than that of any previous ground-based and space-based infrared observatory.

The Observatory was launched with three cryogenically cooled instruments:

- InfraRed Array Camera (IRAC) - PI: Giovanni Fazio, $\mathrm{SAO}^{[2]}$

○ Imaging at 3.6, 4.5, 5.8 and 8.0 microns.

- InfraRed Spectrograph (IRS) - PI: James Houck, Cornell ${ }^{[3]}$

○ Spectroscopy from 5.2 to 38 microns and image at 16 and 22 microns.

- Multiband Imaging Photometer for Spitzer (MIPS) - PI: George Rieke, Arizona ${ }^{[4]}$

○ Imaging at 24, 70 and 160 microns and low-resolution spectroscopy from 55-95 microns.

The cryogenic mission lifetime requirement was two and half years and the observatory exceeded this by more than factor of two. After five and half years of science operations the cryogen was depleted on May 15, 2009. During the cryogenic mission the instruments were operated at temperatures 5-8K. Upon depletion of the cryogen, the instrument chamber warmed to $\sim 27 \mathrm{~K}$. At this new equilibrium temperature, the two shortest wavelength bands of the IRAC instrument (3.6 and 4.5 microns) maintain their cryogenic mission performance levels. The longer wavelength IRAC bands (5.8 and 8.0 microns) and all modes of the MIPS and IRS instruments are no longer usable due to the background at the warmer temperature.

*email: lisa@ipac.caltech.edu

Observatory Operations: Strategies, Processes, and Systems III, edited by

David R. Silva, Alison B. Peck, B. Thomas Soifer, Proc. of SPIE Vol. 7737, 77370L

(C) 2010 SPIE $\cdot$ CCC code: $0277-786 X / 10 / \$ 18 \cdot$ doi: $10.1117 / 12.857827$

Proc. of SPIE Vol. 7737 77370L-1 
Science operations for the warm mission, operating the IRAC 3.6 and 4.5 micron channels, began July 28,2009 and can continue through December 2013 (if funded) using our new warm operations model. The observatory continues to execute cutting edge science and remains NASA's most efficient community observatory with more than $90 \%$ of the time spent executing science observations. We describe here how the warm mission, which on paper has one-sixth of the original capabilities (i.e. half of one instrument) has actually been reinvented as an entirely new, scientifically productive mission operating at a substantially lower cost. While the number of operating instruments has been reduced, the observatory still operates 24/7, 365 days a year. The annual operations budget, including data analysis support for the astronomical community, has been reduced by two-thirds from a level of $\sim \$ 72$ million in FY08 to $\sim \$ 24$ million today.

\section{MISSION OVERVIEW}

\subsection{Cryogenic Operations Overview}

During the cryogenic mission Spitzer executed between 7000-7500 hours per year of science observations. The three instruments were operated serially in campaigns of one-two weeks per instrument in the order IRAC-MIPS-IRS, starting again with IRAC every $\sim 35$ days. Observations were scheduled and sequenced one-week at a time. The observatory operated autonomously, executing the sequences, between DSN contacts which were scheduled approximately every 12 hours. A new master sequence and a week's worth of observations were uplinked each week. Data were downlinked approximately every 12 hours and pipeline processed at the SSC before being released from the archive. Pipeline processed data products were reviewed by a quality analysis team at the SSC prior to release.

General Observer science was selected during annual proposal calls with $750-800$ proposals received per cycle. During the first 2.5 years of the mission $20 \%$ of the observing annual observing time was allocated to Guaranteed Time Observers (GTOs) and $80 \%$ to General Observers (GOs). For the final three years of the cryogenic mission the GTO time was reduced to $15 \%$ and the GO time increased to $85 \%$. Up to $5 \%$ of the GO time annually could be awarded as Director's Discretionary Time (DDT). We supported 200-250 new observing programs annually. Archival Research and Theoretical Research proposals, providing support for data analysis, modeling and theoretical research related to Spitzer were also supported as part of the annual proposal cycles. Data analysis funding was provided to all eligible observers with successful proposals. Additional user community funding for the creation of enhanced data products was provided to Legacy programs, a subset of the GO program. Legacy programs were large, coherent programs that in addition to meeting their own science goals provided substantial legacy value for the astronomical community. The Legacy teams returned enhanced data products to the SSC and the InfraRed Science Archive (IRSA) to be served from the permanent Spitzer archive.

Using the nominal scheduling plan no changes could be made to observations later than 5 weeks prior to execution. The project supported up to ten "high" or "medium" impact targets of opportunity per year. High-impact was defined as requiring execution within 48 hours to one week of approval of the observation. Medium-impact was defined as requiring execution within 1-5 weeks of the approval. During the cryogenic mission the project was able to support 3-4 high impact and 6-7 medium impact observations per year. These could either have been proposed as part of a successful GO program or submitted as a DDT proposal.

\subsection{Initial Warm Mission Planning}

Though the exact date of the cryogen depletion was not know in advance, mid-way through the mission we had confidence that it would last into early 2009. No previous cryogenic mission had estimated cryogen depletion at closer than $5 \%$ to the actual date which for the Spitzer mission was $+/$ - three months. While operating a warm mission was always part of the baseline plan for the project, planning began in earnest two and half years prior to the depletion of the cryogen. At that time we were anticipating a plan that would result in a $50 \%$ reduction in the operating cost. It was clear that greater reductions would have to be made in the science operations than the mission operations because the observatory still operates 24 hours a day, even with just one instrument. The only obvious task that changed for mission operations was that we no longer needed to monitor or manage the cryogen. For science operations, support for the MIPS and IRS instruments would no longer be required. The MIPS/IRS teams at the SSC comprised less than $20 \%$ of the total staffing. Therefore other major changes were required to obtain a substantial reduction in operating costs. 


\section{WARM MISSION PLANNING}

\subsection{Guiding Principles for the Warm Mission}

The warm mission had no formal level 1 requirements but we developed five principles that we adhered to throughout the planning process.

\section{Don't do anything stupid.}

This was already the primary mission rule during cryogenic operations and it has proved invaluable in evaluating options.

\section{Maximize the scientific return of the mission.}

Spitzer exists to do the highest quality science possible. We didn't want to continue operating the observatory if the science program wasn't first rate.

\section{Spitzer is a community observatory.}

As the fourth of NASA's Great Observatories, Spitzer has always executed a community driven science program. The selected observations are peer-reviewed and recommended by the astronomical community.

\section{Minimize the risk to the health and safety of the observatory.}

Changes in mission operations introduced for the warm mission should not add substantial risk to the health and safety of the observatory.

\section{Accept additional risk to science.}

While we did not want to make changes that risked losing the observatory, we did make changes that could have negative impact on science. These would be manifested primarily in terms of scheduling efficiency. We accepted reduced mission and science operations staffing levels that would lead to slower recoveries from anomalies, fewer late changes to schedules, fewer high-impact scheduling opportunities and less direct user support.

\subsection{Essential Features in the Planning Process}

In reviewing the entire planning process for the warm mission, there were two essential features that made what we did a success. These were:

\section{Get Community Input}

The most obvious way to reduce costs was too reduce the number of programs supported at the SSC. With the same number of hours per year available to observe this means we need a smaller number of larger programs. We initially presented a plan to our User's Panel and Oversight Committee suggesting that we call for "Exploration Science" programs with a minimum size of 1000 hours. We formed a Warm Mission steering committee of eight external scientists from multiple disciplines, asked them to form teams to write white papers describing what they would do with $>1000$ hour proposals. We then scheduled a community workshop entitled "Science Opportunities with the Spitzer Warm Mission" in June 2007 where the steering committee presented their white papers and the community discussed them and provided feedback. In addition, we asked the workshop attendees to answer the following questions:

- What are the most important science drivers for a warm Spitzer mission?

- What should be the duration of the warm mission?

- What public 'HDF-style' program should be prepared for the cryo/warm transition period?

- What is the appropriate balance between smaller and larger programs?

- Are ToOs an important component of the warm mission? If yes, at what level?

- Should any science programs be specifically solicited for the warm mission?

- Are there any 'huge' ( $>5000$ hours) projects that should be done? If yes, how should they be selected and organized?

- How does the community participate in science of big projects if not part of the executing teams?

- Can most of the review process be done remotely instead of bringing 100 people to Pasadena annually for a week? 
- Should the review of observing proposals and archival/theory proposals be held at the same time or 6 months out of phase?

The results from the Workshop were substantial. Every team that presented a white paper proposed ideas for exciting science, much of which couldn't be done in the cryogenic mission because during the regular TAC process getting $>$ 1000 hours of time would have been impossible. The community strongly recommended continuing to execute peerreviewed science selected from proposals, as opposed to having a large HDF-like program ready when the cryogen ran out. They embraced the Exploration Science concept but also strongly recommended that we maintain the ability to select and execute small programs. Based specifically on feedback from the extrasolar planet community we reduced the minimum size for exploration science proposals from 1000 to 500 hours. There was no justification for changing the annual nature of the proposal process but no strong feeling against trying to make it less expensive by doing more of it remotely instead of all as an in-person review. Though the Spitzer project provided suggested overall plans and changes the community had a substantial impact on the details of how the warm mission operations actually look. The proceedings ${ }^{[5]}$ of the workshop were published and made available to the entire astronomical community.

\section{Examine all mission and science operations functions.}

a. What do we no longer need to do?

b. Can we merge teams?

c. What are we doing right that we shouldn't change?

d. What is "good enough?"

While engaging the community was crucial for getting guidance on how to craft and new science program that was still very high quality but less expensive to execute, the internal process for every team on the project was equally important. Each team examined what they did and tried to determine what was critical to keep doing and shouldn't change, what needed to be kept but could be modified to be less labor intensive, and what could be dropped. This was done keeping in mind the five guiding principles listed in section 3.1. Examples of things that changed, and things that didn't are provided in section 4 .

\section{CHANGES MADE FOR THE WARM MISSION}

Below we provide a summary of the highlights of things that changed between the cryogen and warm missions.

\section{Reduced the number of observing programs supported annually from $\sim 250$ to $\sim 60$.}

Impact to the Community: While fewer individual programs are supported, the introduction of Exploration Science programs with a minimum size of 500 hours, enabled entirely new scientific investigations that were not possible during the cryogenic mission where the maximum time awarded to a single proposal during a typical GO cycle was 500 hours.

Impact to the Project: Allowed for reduction in User Support staff and allowed us to substantially reduce costs for the review since instead of receiving $\sim 800$ proposals a year we now receive $\sim 150$. The changes to the review process are discussed in the next item.

\section{Changed the panel review portion of the proposal peer review into telecons instead of face-to-face meetings.}

Impact to Community: Review panelists don't have to travel to the review meeting. The panel reviews are held as half-day telecons and the Time Allocation Committee (made up of the panel chairs) meeting is held two weeks after the panels as a one-day face-to-face meeting.

Impact to the Project: The annual cryogenic mission proposal review meetings involved bringing $\sim 100$ external reviewers to Pasadena for 3-6 days to review the proposals. The direct costs to support this were $\$ 250-300 \mathrm{k}$ annually. The meetings required the support of $\sim 35$ people from the SSC. With the smaller number of proposals the panel reviews can be handled as half-day telecons and then direct expenses are only required for bringing the TAC out for a 
one-day meeting to recommend the large proposals. This costs $\sim \$ 30 \mathrm{k}$, so it is an order of magnitude reduction in the cost of the review. In addition, the entire review requires the support of $\sim 7$ people at the SSC.

\section{Project will only support one high-impact scheduling interrupt per year and the request must be made via a DDT proposal.}

Impact to the Community: High and medium-impact target of opportunity requests can no longer be made in GO proposals. This is lost as a general user capability. The observatory will attempt to support a truly scientifically worthy observation that requires a very quick turn-around.

Impact to the Project: Allows for reductions in mission and science operations personnel.

\section{No late changes to schedules except for observatory health and safety.}

Impact to the Community: During the cryogenic mission, late changes to observations that were already scheduled, were often supported to improve the science. Changes to an already approved schedule were supported by creating a 'Pass 2' of the schedule products. If an observer discovered an error in their coordinates or observing strategy, even though it was against the formal rules to modify the sequences after scheduling, if it could be supported the changes would be made to the schedule. During the warm mission, resources are not available to do this. If an observer discovers their coordinates are wrong after Pass 1 has been approved then the wrong targets will be observed.

Impact to the Project: Allows for reduction in scheduling support staff in both mission and science operations.

\section{Add one week to the front-end of the scheduling process at the SSC.}

Impact to the Community: Any changes to observing requests must be made at least six weeks prior to execution instead of five weeks.

Impact to the Project: The extra week in the early scheduling process allow the SSC Operations Planning and Scheduling Team (OPST) to better accommodate complicated constraints that cross weekly scheduling. The project looked closely at the cadence for building schedules and elected to not change building one-week at a time. There were no real efficiencies to be gained by changing the well-tested processes and procedures used during the cryogenic mission.

\section{Schedule downlink opportunities every 24 hours instead of every 12 hours.}

Impact to the Community: Little direct impact. Provides slightly more observing time since the overhead of slewing to earth-point only happens once per day.

Impact to the Project: Allows reduction of $\sim 50 \%$ in Flight Control staffing and reductions in other mission operations staff that support the spacecraft contacts. Downlinks every 24 hours instead of every 12 hours reduces flexibility in the scheduling of high data volume programs and reduces the number of opportunities for uplinking the weekly schedules. Fewer regularly scheduled downlinks also complicates scheduling heavily constrained, long exoplanet observations. The data volume for the 3.6 and 4.5 micron IRAC channels is about $60 \%$ of the volume for all four channels, so operating warm doesn't quite halve the typical data volume. Fewer downlink opportunities also increases the risk of observations being skipped if multiple downlinks are missed and the mass memory card becomes full. The schedules are designed to be single fault tolerant, i.e. we can miss any one downlink pass without requiring the schedule to be rebuilt.

\section{All software, except pipelines and data analysis tools, is effectively frozen.}

Impact to the Community: The observation planning software and the mission operations tools are mature, so this has minimal direct impact on the community. The community is impacted by reduced development on the data processing tools. 
Impact to the Project: Mission operations reduced levels of multi-mission and institutional infrastructure to a minimum and keep minimal support for sustaining and maintenance. The SSC froze the uplink and scheduling software and continues with a minimal amount of development support for the IRAC pipelines and data analysis tools.

\section{Reduced engineering staff for performance analysis and anomaly response.}

Impact to the Community: Anomalies will take longer to recover from and therefore more science time will be lost.

Impact to the Project: Same.

\section{SSC dropped Data Quality Analysis Review}

Impact to the Community: The SSC stopped doing data quality analysis (DQA) review of the pipeline processed data prior to it being archived. During the cryo mission, the DQA team reviewed all of the data and occasionally found issues that were then addressed by the instrument team prior to releasing the data. The DQA check has effectively been moved to the observer. Problems are rarely found so this should have a small impact on the community.

Impact to the Project: Reduction of two FTEs at the SSC.

\section{Ended the Spitzer Fellowship Program}

Impact to the Community: This would have been a loss of five post-doctoral fellowships per year but NASA realigned the Great Observatory fellowship programs in 2009. The Hubble Fellowship program was increased by five fellowships annually and they now support all science related to missions supporting NASA's Cosmic Origins theme, which includes Spitzer.

Impact to the Project: Reduced User Support and Community Affairs staffing.

\section{Reduced Data Analysis Funding to the Community}

Impact to the Community: During the cryogenic mission Spitzer annually provided nearly $\$ 30$ million in data analysis funds to successful GO investigators and to the GTOs. The "Spitzer Constant" (the average dollar/hour of observing time) was $\$ 3000$ to $\$ 4000$. The User Community budget has been reduced to $\$ 3-4$ million/year for the warm mission. Programs less than 20 hours now receive now direct funding and the Spitzer constant is $\sim \$ 700$.

Impact to the Project: Fewer funding contracts to issue and manage.

\section{Stopped soliciting Archival and Theoretical Research Programs}

Impact to the Community: Spitzer archival research is now supported through NASA's ADP program. Theoretical research programs must be submitted to other NASA programs. Spitzer issued \$2-2.5 million per year in archival and theoretical research funding.

Impact to the Project: Fewer proposals to support during the annual cycles and fewer funding contracts to issue and manage.

\section{Reduced SSC Direct User Support Staffing}

Impact to the Community: Increased response time for Helpdesk questions. SSC stopped hosting Data Analysis Workshops and reduced participation at AAS and other science meetings.

Impact to the Project: Reduced SSC staff level.

\section{Halved the SSC Public Affairs/Outreach Staff}


Impact to the Community: Little impact for the professional astronomical community but several outreach activities were curtailed or dropped.

Impact to the Project: Reduced SSC outreach staff by $50 \%$. Additional funding was provided separately by NASA, to IPAC, to support what was previously the Spitzer Teacher's program.

\section{COMPARISON OF CRYOGEN AND WARM MISSION}

Table 1 provides a comparison of the staffing levels for different components of the Spitzer project throughout the mission. The three components of the project that are compared are:

1. Project Offices: The project management, science and assurance offices at Jet Propulsion Laboratory (JPL).

2. Mission Operations System (MOS)

The MOS includes the mission operations staff at the Jet Propulsion Laboratory (JPL), as well as the Observatory Engineering Team (OET) at Lockheed, Denver. The MOS is responsible for the health and safety of the observatory, flight operations, mission sequencing and initial processing of the science data into FITS files.

3. Spitzer Science Center (SSC), Caltech

The SSC is responsible for science operations of the observatory including instrument support, proposal selection, scheduling, data processing and user support. The SSC is the primary interface to the science community and also provides Public Affairs and Education and Public Outreach.

The three time points compared are:

1. Start of science operations (December 2003). Nominal science operations began December 1, 2003. The mission operations staff is still ramping down at this point from the peak launch levels. The science operations staff is also still at peak level but is closer to the steady-state cryogen operations level.

2. Cryogenic mission mid-point (FY06). The operations are mature and the total staffing is $\sim 73 \%$ of that at the start of nominal science operations. Mission operations decreased 45\% from the December 2003 level and science operations decreased $15 \%$.

3. Warm mission (FY10). The total project staffing is now $32 \%$ of the staffing at the start of nominal science operations. Compared to the staffing for the cryogenic mission, mission operations has been reduced by more than a third and science operations by two-thirds. Of the 62 FTE decrease for science operations, only about 15 FTEs were supporting the IRS and MIPS instruments that don't operate in the warm mission.

\begin{tabular}{|c|c|c|c|}
\hline \multicolumn{4}{|c|}{ Spitzer Project - Mean FTEs / year } \\
\hline & $\begin{array}{l}1 \text { Dec } 2003 \\
\text { Start of } \\
\text { Science } \\
\text { Operations }\end{array}$ & $\begin{array}{c}\text { FY06 } \\
\text { Cryogen } \\
\text { Operations }\end{array}$ & $\begin{array}{c}\text { FY10 } \\
\text { Warm } \\
\text { Operations }\end{array}$ \\
\hline Project Offices & 15.8 & 9.0 & 5.7 \\
\hline Mission Operations & 64.7 & 35.4 & 22.5 \\
\hline SSC + Outreach & 112.5 & 95.8 & 34.0 \\
\hline Total & 193.0 & 140.2 & 62.2 \\
\hline \multicolumn{4}{|c|}{$\%$ FTEs compared to Start of Science Ops } \\
\hline & & $73 \%$ & $32 \%$ \\
\hline
\end{tabular}

Table 1. Comparison of project staffing levels at the start of science operations, the middle of the cryogen mission, and the current warm mission levels. 
Table 2 provides comparisons of several quantities, other than staffing, between the warm and cryogenic operations. The observatory remains very efficient in terms of the number of hours of science scheduled, but has substantially reduced the number of programs supported. The operating budget has been decreased by $\sim 45 \%$ and user community funding has been decreased by an order of magnitude. The mean operating cost for each hour of science is $<\$ 3000$.

\begin{tabular}{|l|c|c|}
\hline & $\begin{array}{l}\text { Cryogen } \\
\text { Operations }\end{array}$ & $\begin{array}{l}\text { Warm } \\
\text { Operations }\end{array}$ \\
\hline $\begin{array}{l}\text { Observing Programs } \\
\text { Supported Annually }\end{array}$ & 250 & 60 \\
\hline $\begin{array}{l}\text { Mean Hours of Science } \\
\text { Executed Annually }\end{array}$ & 7250 & 7500 \\
\hline $\begin{array}{l}\text { Operating Budget } \\
\text { (\$million) }\end{array}$ & 37 & 21 \\
\hline $\begin{array}{l}\text { User Community Budget } \\
\text { (\$million) }\end{array}$ & 35 & 3 \\
\hline Total Budget (\$million) & 72 & 24 \\
\hline Mean Operating Cost/Hour & $\$ 5,100$ & $\$ 2,800$ \\
\hline
\end{tabular}

Table 2. Comparison of science program and operating costs for the cryogen and warm mission phases.

\section{PUBLICATION STATISTICS}

Figure 1 shows publication statistics for several NASA missions. The counts for all the missions were made by the SSC using the same methodology for each mission ${ }^{[6]}$. In 2009, Spitzer had a comparable number of refereed publications as the Hubble Space Telescope. To predict the potential scientific productivity of Spitzer with just the warm IRAC instrument, we examined how many of the current publications utilized IRAC data.

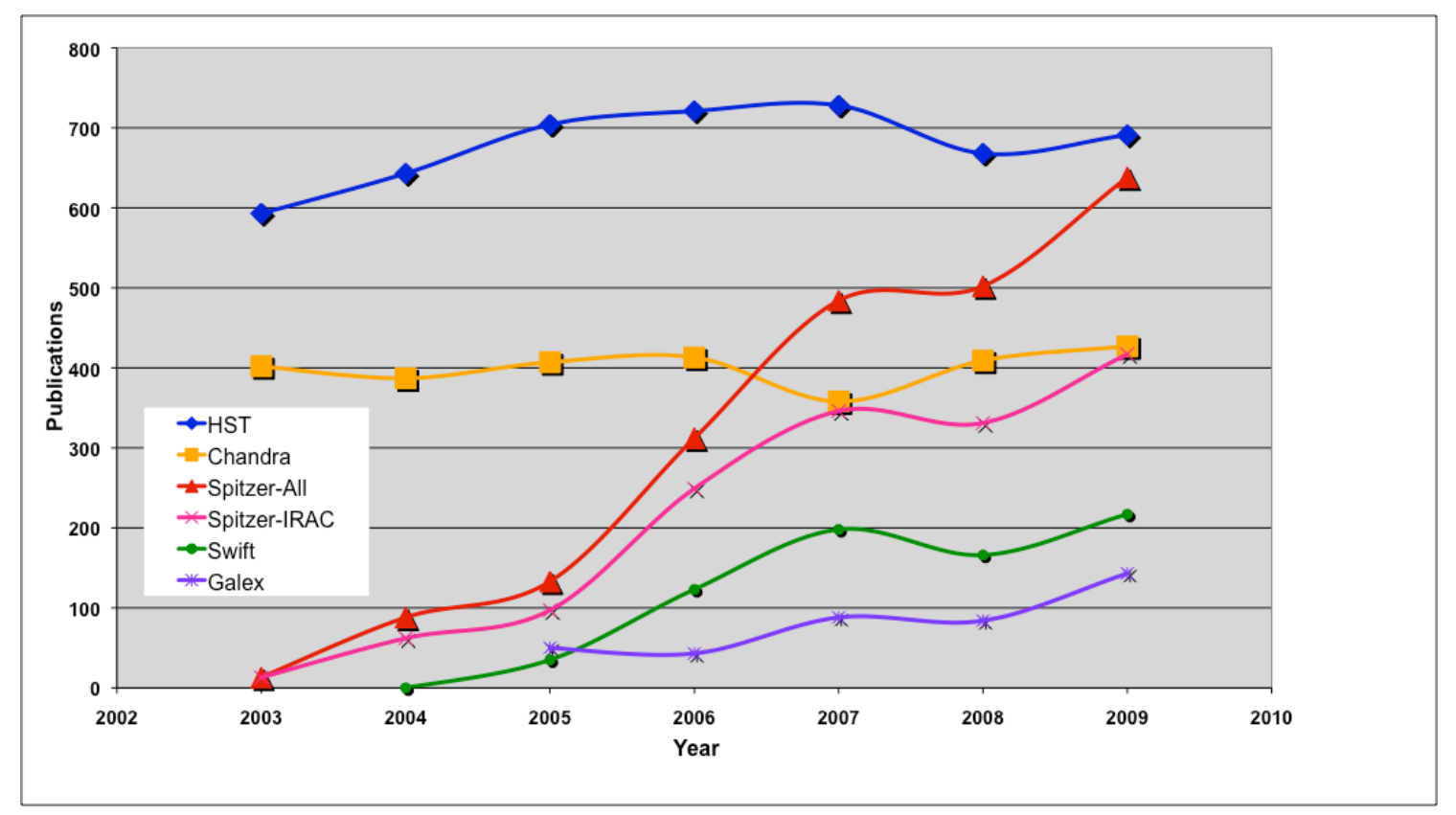

Figure 1. Refereed publication statistics for several NASA missions as counted by the SSC. The counts for the entire Spitzer mission are shown in red and the subset of all of those publications that used data from the IRAC are shown in pink. 
The results are striking. The IRAC instrument was on for $27 \%$ of the time during the cryogenic mission and yet the data is used in $66 \%$ of the refereed papers. The IRAC publication counts are shown in figure 1 in pink. Even though the warm mission supports fewer individual programs, the amount of IRAC data gathered will be at least 2 times greater annually than we supported in the cryogen mission. We have every expectation that Spitzer operating warm will continue to be a highly productive mission.

\section{SUMMARY}

The Spitzer Space Telescope has successfully transitioned from the cryogen mission to the IRAC warm mission. The observatory is operating a rich, diverse science program that is driven by the creativity of the science community. The warm mission science addresses the most compelling questions of current day astrophysics, ranging from probing the atmospheric structure of exoplanets to determining when the first galaxies formed. With only two arrays operating, Spitzer still produces a scientific return comparable to that of the cryogenic mission in terms of data volume and number of observations executed. The project cost has been reduced to one-third of the level during the cryogenic mission and we believe that Spitzer is producing science comparable to multiple Explorer missions.

\section{REFERENCES}

[1] Werner, M., Roellig, T., Low, F., Rieke, G., Rieke, M., Hoffmann, W., Young, E., Houck, J., Brandl, B., Fazio, G., Hora, J., Gehrz, R., Helou, G., Soifer, B., Stauffer, J., Keene, J., Eisenhardt, P., Gallagher, D., Gautier, T., Irace, W., Lawrence, C., Simmons, L., Van Cleve, J., Jura, M., Wright, E., 2004, ApJS, 154, 1.

[2] Fazio, G. G., Hora, J. L., Allen, L. E., Ashby, M. L. N., Barmby, P., Deutsch, L. K., Huang, J.-S., Kleiner, S., Marengo, M., Megeath, S. T., Melnick, G. J., Pahre, M. A., Patten, B. M., Polizotti, J., Smith, H. A., Taylor, R. S., Wang, Z., Willner, S. P., Hoffmann, W. F., Pipher, J. L., Forrest, W. J., McMurty, C. W., McCreight, C. R., McKelvey, M. E., McMurray, R. E., Koch, D. G., Moseley, S. H., Arendt, R. G., Mentzell, J. E., Marx, C. T., Losch, P., Mayman, P., Eichhorn, W., Krebs, D., Jhabvala, M., Gezari, D. Y., Fixsen, D. J., Flores, J., Shakoorzadeh, K., Jungo, R., Hakun, C., Workman, L., Karpati, G., Kichak, R., Whitley, R., Mann, S., Tollestrup, E. V., Eisenhardt, P., Stern, D., Gorjian, V., Bhattacharya, B., Carey, S., Nelson, B. O., Glaccum, W. J., Lacy, M., Lowrance, P. J., Laine, S., Reach, W. T., Stauffer, J. A., Surace, J. A., Wilson, G., Wright, E. L., Hoffman, A., Domingo, G., Cohen, M., The Infrared Array Camera (IRAC) for the Spitzer Space Telescope, 2004, ApJS, 154, 10.

[3] Houck, J., Roellig, T., van Cleve, J., Forrest, W., Herter, T., Lawrence, C., Matthews, K., Reitsema, H., Soifer, B., Watson, D., Weedman, D., Huisjen, M., Troeltzsch, J., Barry, D., Bernard-Salas, J., Blacken, C., Brandl, B., Charmandaris, V., Devost, D., Gull, G., Hall, P., Henderson, C., Higdon, S., Pirger, B., Schoenwald, J., Sloan, G., Uchida, K., Appleton, P., Armus, L., Burgdorf, M., Fajardo-Acosta, S., Grillmair, C., Ingalls, J., Morris, P., Teplitz, H., The Infrared Spectrograph on the Spitzer Space Telescope, 2004, ApJS, 154, 18.

[4] Rieke, G., and E. T. Young, C. W. Engelbracht, D. M. Kelly, F. J. Low, E. E. Haller, J. W. Beeman, K. D. Gordon, J. A. Stansberry, K. A. Misselt, J. Cadien, J. E. Morrison, W. B. Latter, A. Noriega-Crespo, D. L. Padgett, K. R. Stapelfeldt, D. C. Hines, E. Egami, J. Muzerolle, A. Alonso-Herrero, M. Blaylock, H. Dole, J. L. Hinz, E. Le Floc'h, C. Papovich, P. G. Perez-Gonzalez, P. S. Smith, K. Y. L. Su, L. Bennett, D. T. Frayer, D. Henderson, N. Lu, F. Masci, M. Pesenson, L. Rebull, J. Rho, J. Keene, S. Stolovy, S. Wachter, W. Wheaton, P. L. Richards, The Multiband Imaging Photometer for Spitzer, 2004, ApJS, 154, 25.

[5] Storrie-Lombardi, L. J., and Silbermann, N. A., "The Science Opportunities of the Warm Spitzer Mission Workshop," AIP Conference Proceedings 943; Conference Location and Date: Pasadena, California, 4-5 June 2007.

[6] Scire, E., Chan, B. H., Silbermann, N., Shields, A., "The Spitzer bibliography database: bibliographic statistics," Proc. SPIE 7737:70 (2010). 